\title{
Multiple free electron laser pulse illumination of a carbon coated silicon substrate
}

Björn Siemer, Tim Hoger, Marco Rutkowski, Martina Menneken, Stefan Düsterer, et al.

Björn Siemer, Tim Hoger, Marco Rutkowski, Martina Menneken, Stefan Düsterer, Helmut Zacharias, "Multiple free electron laser pulse illumination of a carbon coated silicon substrate," Proc. SPIE 8777, Damage to VUV, EUV, and X-ray Optics IV; and EUV and X-ray Optics: Synergy between Laboratory and Space III, 87770F (3 May 2013); doi: 10.1117/12.2017248 


\title{
Multiple free electron laser pulse illumination of a carbon coated silicon substrate
}

\author{
Björn Siemer*a, Tim Hoger ${ }^{\mathrm{a}}$, Marco Rutkowski ${ }^{\mathrm{a}}$, Martina Menneken ${ }^{\mathrm{b}}$, Stefan Düsterer ${ }^{\mathrm{c}}$, \\ Helmut Zacharias ${ }^{\mathrm{a}}$ \\ ${ }^{a}$ Westfälische Wilhelms-Universität Münster, Physikalisches Institut, Corrensstrasse 24, \\ 48149 Münster, Germany; \\ ${ }^{b}$ Westfälische Wilhelms-Universität Münster, Institut für Mineralogie, Wilhelm-Klemm-Str. 10, \\ 48149 Münster, Germany; \\ ${ }^{\mathrm{c}}$ Deutsches Elektronen-Synchrotron, HASYLAB, Notkestr. 85, 22607 Hamburg Germany \\ *b.siemer@uni-muenster.de; phone +49 2518339 109; fax +49 2518333 604; uni-muenster.de/Physik.PI/Zacharias/
}

\begin{abstract}
The fourth generation of XUV-, soft x-ray- and x-ray-light sources, like the free electron lasers FLASH and FERMI@Elettra, leads to new seminal scientific findings and technical challenges. For the facilities the question of the beam transport is of utmost importance. To provide a good reflectivity over a large range of photon energies up to about $300 \mathrm{eV}$ mostly carbon coated silicon mirrors illuminated under gracing incidence angle are mostly chosen. Thereby the coating for the mirrors must tolerate high light intensities at high photon energies and also high repetition rates. In the present experiment an amorphous carbon coated silicon substrate was illuminated at photon energies of $21 \mathrm{~nm}(58 \mathrm{eV})$ and an average pulse energy of $\sim 27 \mu \mathrm{J}$. The ellipsoidal spot size of $300 \mu \mathrm{m} \times 600 \mu \mathrm{m}$ at FLASH leads to a fluence of $0.019 \mathrm{~J} / \mathrm{cm}^{2}$. The influence of multiple $(100-20.000)$ light pulses to the coated surface is analyzed. Depending on the number of pulses a change in reflectivity is visible under a light microscope. Both an AFM profile and measurements with a profilometer yield no topological changes. The investigation of the illuminated spots with a microfocus Raman spectrometer shows a decrease of the carbon signal at higher pulse repetition rates.
\end{abstract}

Keywords: FLASH, carbon coatings, XUV optics, multiple illuminations

\section{INTRODUCTION}

In the last years the popularity of fourth generation light sources, so-called free electron lasers, grows incredibly due to new findings in a wide range of fundamental physics. The first facility the free electron laser (FEL) in Hamburg (FLASH) started with the generation of XUV- and soft x-ray light in 2005 (25 to $300 \mathrm{eV}$ ) [1]. A similar photon energy range is covered by the FEL FERMI @ Elettra [2]. During their first years of operation the FELs provide light for investigations in atoms, ions, molecules and clusters, creation and characterization of warm dense matter, diffraction imaging of nanoparticles, spectroscopy of bulk solids and surfaces, investigation of surface reactions and spin dynamics [see [3] and references therein]. Additionally, a lot of effort was achieved to develop new photon diagnostic equipment and investigate new experimental techniques customized for the use at FEL facilities. To enable users a continuous good beam quality the facilities invest significant efforts for the transportation of radiation. One of the main components for a possible change in the beam quality after the generation of the light is due to intensity loss on optical equipment. At FLASH carbon coated mirrors are used to guide the XUV beam to the required beamline. To avoid damage due to high power of FLASH pulses and to provide high reflectivity grazing incidence angles of $\alpha \sim 3^{\circ}$ are chosen. The mirrors consist mostly of silicon substrates providing a sufficient heat transport. The substrates are coated with carbon with a surface roughness less than $5 \AA$ over the whole surface [3]. Carbon has a high reflectivity over the wavelength range of the provided FLASH pulses ( 25 to $300 \mathrm{eV}$ ). For carbon coated silicon mirrors the reflectivity is shown in figure 1 (a) for an incidence angle of $3^{\circ}, 6^{\circ}$ and $80^{\circ}$ for the p-polarized FLASH radiation. Thereby the reflectivity curve for an angle of $80^{\circ}$ reflects the presented experimental conditions.

Damage to VUV, EUV, and X-ray Optics IV; and EUV and X-ray Optics: Synergy between Laboratory and Space III, edited by Libor Juha, Saša Bajt, Richard London, René Hudec, Ladislav Pina, Proc. of SPIE Vol. 8777, 87770F

(c) 2013 SPIE · CCC code: 0277-786X/13/\$18 - doi: 10.1117/12.2017248 


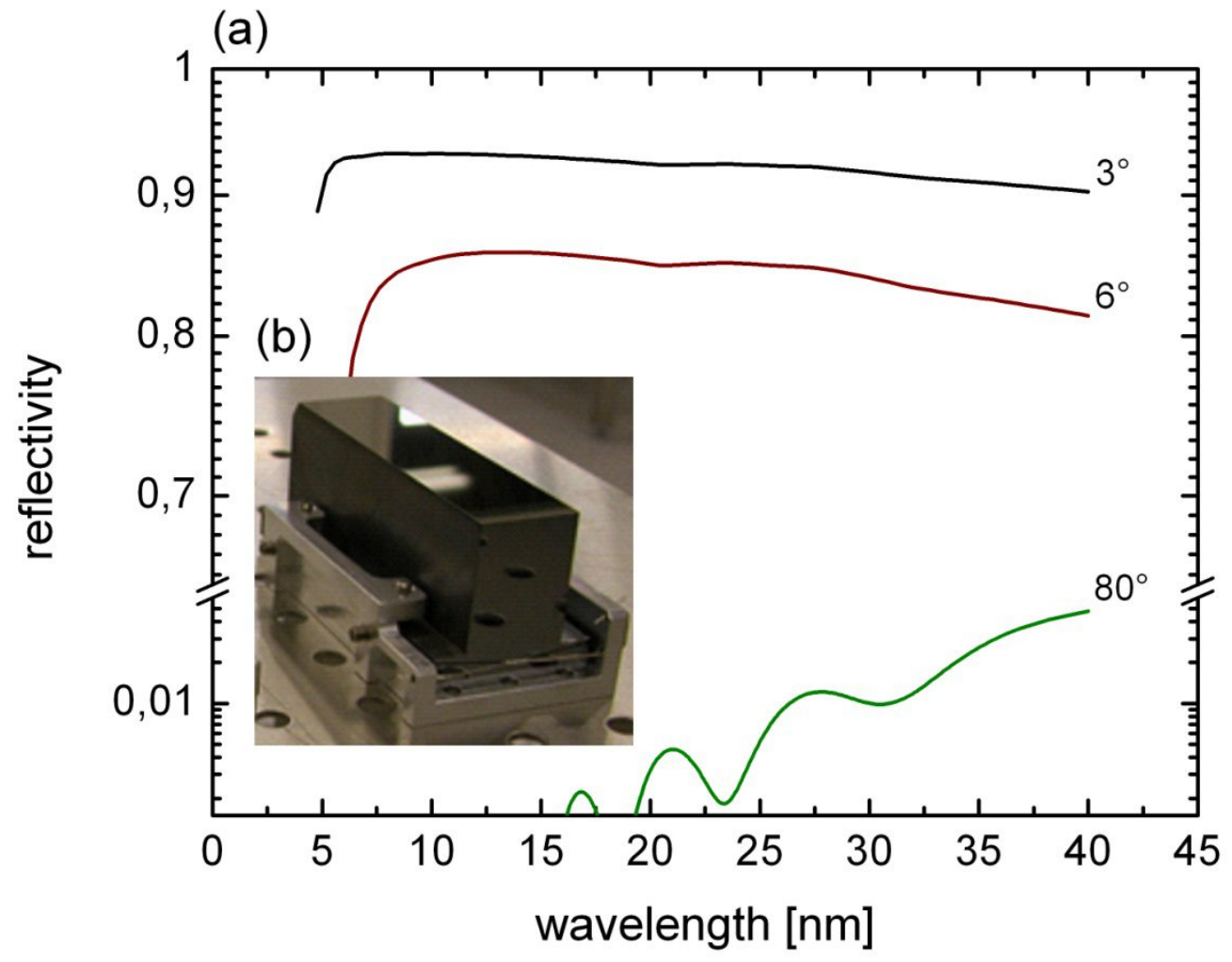

Figure 1. (a) The reflectivity curve for carbon coated mirrors at three different angles of grazing incidence as a function of the wavelength [4]. (b) A picture of a carbon coated silicon substrate mounted in a mirror holder. This mirror is used in the split and delay unit (SDU) integrated at BL2 at FLASH.

To avoid jitter-free XUV-pump and XUV-probe experiments at FLASH as well as to characterize the temporal structure of its high power pulses a novel split- and delay unit (SDU) has been constructed for the VUV, XUV and soft X-ray spectral region. Applying geometrical wave front beam splitting and an all-reflective optical design with grazing incidence angles of $3^{\circ}$ and $6^{\circ}$ at the mirrors the instrument is capable to handle the high power FLASH pulses with exceptional high efficiency up to $300 \mathrm{eV}$ [5]. This SDU is a installed on BL2 at FLASH and applied to time-resolved measurements in atomic and cluster physics, as well as to determine the temporal structure of the FLASH pulses [6-12]. A typical carbon coated mirror substrate is shown in the inset of figure 1 (b). The T shaped substrate enables an optimal mounting on the mirror holders. The length of the substrate is $100 \mathrm{~mm}$ in length with an $25 \mathrm{~mm}$ wide optical plain level. On the top side the flat carbon coated mirror surface is visible with an assured roughness of $\sim 0.2 \mathrm{~nm}$.

In previous single shot experiments damage thresholds were conducted at FLASH operating at a wavelength of $32.5 \mathrm{~nm}$ (38 eV) using samples of $\mathrm{B}_{4} \mathrm{C}$, amorphous $\mathrm{C}(\mathrm{a}-\mathrm{C})$, and chemical vapor deposited diamond, $\mathrm{Si}$, and $\mathrm{SiC}$ exposed to fluences of up to $2.2 \mathrm{~J} / \mathrm{cm}^{2}$ [13]. A found single-pulse damage threshold for the materials is comparable to the conventional melt threshold. For fluences less than $200 \mu \mathrm{J} / \mathrm{cm}^{2}$ after the radiation of the a-C sample only extrusions were observed. The damage threshold found for the amorphous carbon was $60 \mu \mathrm{J} / \mathrm{cm}^{2}$. The calculated melt thresholds for the above surface coatings except for a-C yield values between $40-180 \mu \mathrm{J} / \mathrm{cm}^{2}$. For the a-C coating only extrusions were observed. In later experiments the damage threshold of a $40 \mathrm{~nm}$ thick a-C coated mirror was determined to $43.7 \pm 13 \mathrm{~mJ} / \mathrm{cm}^{2}$ for a FLASH wavelength of $7 \mathrm{~nm}(177 \mathrm{eV})[14]$. In further experiments to analyze the behavior of mirrors under multiple laser shots an a-C coated $\mathrm{Si}$ substrate was irradiated with $46.9 \mathrm{~nm}$ laser pulses [15]. As light source a Ne-like $\mathrm{Ar}^{8+}$ XUV laser with a pulse duration of $1.7 \mathrm{~ns}$ was applied. At that wavelength a damage threshold fluence of $1.1 \mathrm{~J} / \mathrm{cm}^{2}$ was determined for the single shot exposure. For multiple sample illuminations a fluence of $500 \mathrm{~mJ} / \mathrm{cm}^{2}$ was applied with a rate of 10 to 40 shots. They observed a damage of the a-C layers exposed to the shots at a 
fluence of about half the single shot damage threshold. Furthermore, no structural change of the a-C layer by Raman microscopy was observed. These findings support the key role of non-thermal processes in damaging a-C mirror coatings for the new light sources even if the intensity does not reach the single shot damage threshold.

\section{EXPERIMENTAL SET UP}

\subsection{UHV system}

To illuminate the carbon coated Si surface an existing experimental set up was extended. Normally the set up is used for surface science experiments in the laboratory and at light sources like FLASH [7,8]. In order to avoid damage on the carbon coatings of the original Si mirror substrates an analogous substrate was coated in the same mirror coating process. Small square-cut silicon substrates with a length of $1 \mathrm{~cm}$ and a thickness of $1 \mathrm{~mm}$ were used. With this procedure a similar coating like on the mirror substrates could be assured. The $45 \pm 5 \mathrm{~nm}$ thick amorphous carbon coating with a roughness of about $0.2 \mathrm{~nm}$ was realized by magneton sputtering (GKSS Geesthacht). These substrates were placed in an UHV chamber which was evacuated by an oil free pump system to a base pressure of $7 \times 10^{-10}$ Torr. For an easy handling the sample was attached on a sample holder on an X-Y-Z stage with a $360^{\circ}$ rotatable feed through. The X-Y-Z stage was movable by step motors controlled by a computer software based on Labview. Due to a liquid nitrogen containing reservoir the sample holder is cooled to $95 \mathrm{~K}$ as monitored with a k-type thermocouple attached to the surface. For an optimal temperature contact the Si substrate was connected to the sample holder via silver conduction paste.

\subsection{FLASH properties}

The experiment was performed at beamline 1 at FLASH providing a weakly focused spotsize in the focal point of about $100 \mu \mathrm{m}$ diameter. The sample is placed outside the focal point to permit a lower fluence. Due to the placement an ellipsoidal spot size of about $300 \mu \mathrm{m}$ × $600 \mu \mathrm{m}$ was obtained, because beam strikes the analyzed surface at an angle of incidence of $\vartheta=10^{\circ}$ relative to the surface normal. FLASH operated at a wavelength of $\lambda=21 \mathrm{~nm}(\mathrm{~h} v=58 \mathrm{eV})$ in a $5 \mathrm{~Hz}$ single bunch mode with a spectral energy width of about $0.8 \mathrm{eV}$. The averaged pulse energy of $27 \pm 3 \mu \mathrm{J}$ detected by the gas monitor detector (GMD) [16] resulted in an averaged fluence of $19 \mathrm{~mJ} / \mathrm{cm}^{2}$ applied to the surfaces. The p-polarized FLASH light had an averaged pulse duration of about $30 \mathrm{fs}$ [6]. The pulse tag provided by FLASH was recorded and later combined with the recorded GMD signal. To investigate the behavior of the carbon coating under multiple pulse illumination a raster of spots was planned. The sample was adjusted for the illumination and after a defined number of pulses the sample holder was moved to the next position. The moving procedure was fully automated via Labview software and takes less a second for one movement.

\section{RESULTS AND DISCUSSION}

\subsection{Optical microscopy}

A fist analysis of the irradiated coating was performed with an optical microscope. Figure 2 (a) shows several spots with a high illumination number of 20.000 FLASH pulses, figure 2 (b) shows spots with 100 pulses (not visible) to 10.000 consecutive pulses. A change in optical reflectivity is evident depending on the number of pulses applied. Furthermore, a change in the spot size can be identified. The spots with less illumination are clearly smaller than those at higher exposures. 

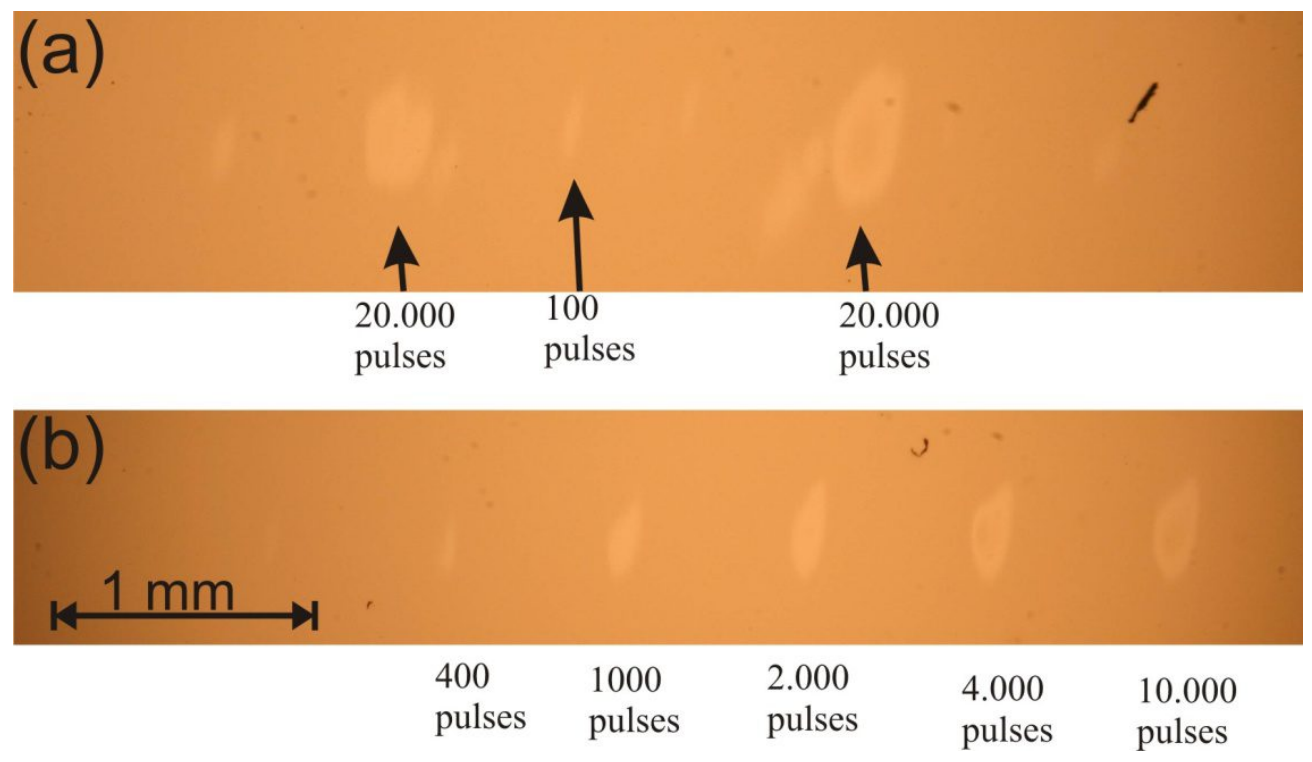

Figure 2. Sequence of multiple exposures on a carbon coated silicon surface under an optical microscope. The number of pulses varies from 100 to 20.000 . Under the optical microscope a change in reflectivity depending on the exposed number of laser pulses is visible.

\subsection{Profilometry}

The previous and afterwards realized investigations of the illuminated surface with an atomic force microscope (AFM, ) was without any result since the scan area of the microscope is relatively small compared to the beam diameter. Therefore an optical profiler (Veeco, NT3300) was employed which allows a closer view to the surface conditions. In figure 3 a scan with the profilometer of the investigated surface is shown. In this case the area of interest is amounts to about $0.9 \times 1.2 \mathrm{~mm}^{2}$. The area on the carbon coated silicon surface exposed to 20.000 FLASH pulses is indicated by the ellipse. Over the whole image the roughness is $\pm 15 \mathrm{~nm}$ in height, as indicated by color code. The weakly roughness is possible caused by the imprecise surface of the used silicon substrates. At the position of the illuminated spot no obvious change in the surface profile caused by the irradiation can be recognized.

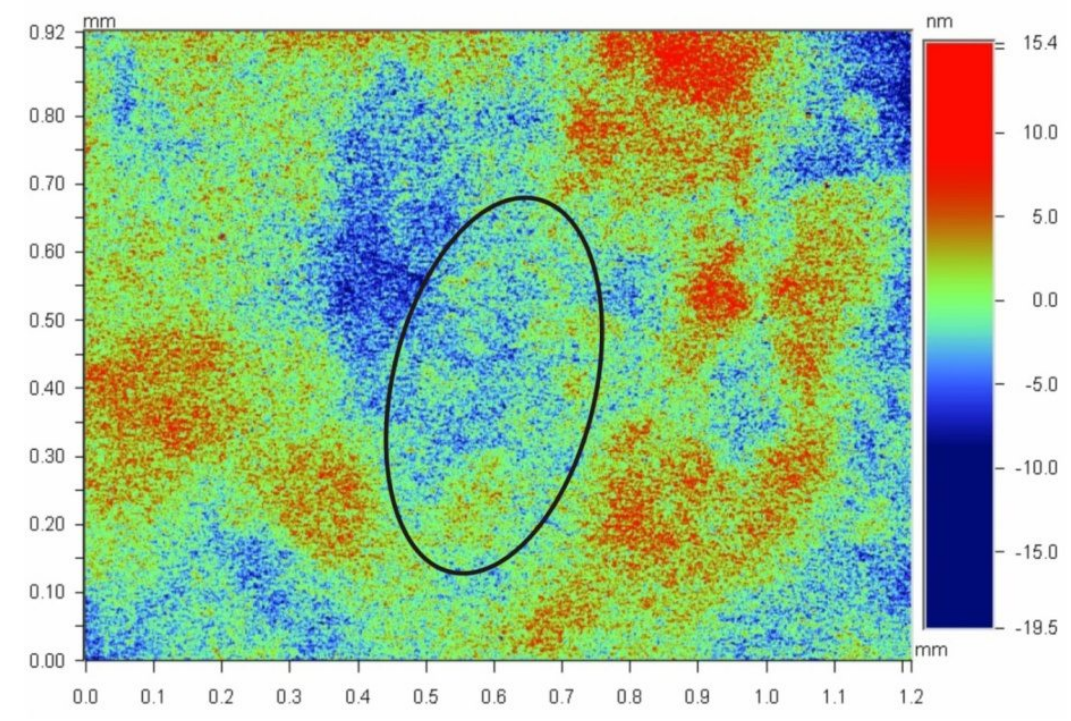

Figure 3. Image of the illuminated carbon coated silicon surface taken with a profilometer. The black ellipse marks the spot which was illuminated by 20.000 FLASH pulses. 


\subsection{Raman spectrometry}

The observed optical change in reflectivity can also be caused by a possible material or structural change in the carbon surface. To determine the changes a micro Raman spectrum has been taken for every different spot with a dispersive Raman spectrometer (Jobin Yvon HR800). The $532 \mathrm{~nm}$ line of a $14 \mathrm{~mW}$ Nd-YAG laser is used for excitation and a grating of 1.800 grooves $/ \mathrm{mm}$ with a spectral resolution of $1.1 \mathrm{~cm}^{-1}$ (near $1.330 \mathrm{~cm}^{-1}$ ) for detection.

In figure 4 (a) the taken Raman spectra are shown. The red line indicates the spectra of the unirradiated carbon coated surface while the black line indicates the pure unirradiated silicon. The lines between belong to the sequence of illumination. The G peak at about $1.600 \mathrm{~cm}^{-1}$ is a good indicator of disordered carbon while the D peak at around $1.350 \mathrm{~cm}^{-1}$, is strongest for ordered carbons. The T peak lying around $980 \mathrm{~cm}^{-1}$ indicates hydrogenated carbons [17]. With a higher number of irradiated pulses a decrease of the carbon signal ( $G$ and $D$ peak) is visible. Also a decrease of the intensities of the $G$ peak relative to the $D$ peak is observable. Integration of the two peaks leads to the intensities shown in figure 4 (b). The black dots represent the integral of the carbon peaks in the Raman spectra plotted against the number of pulses illuminating the surface. The two data points, placed at pulse number 20, represent the Raman signal on the unirradiated and the pure silicon surface, respectively. The data show a decrease of the carbon signal from the illumination with 100 shots to a nearly constant value at a number of pulses higher than 2000 . Similar to this finding the signal of the silicon peak increase for higher pulse number hitting the surface.

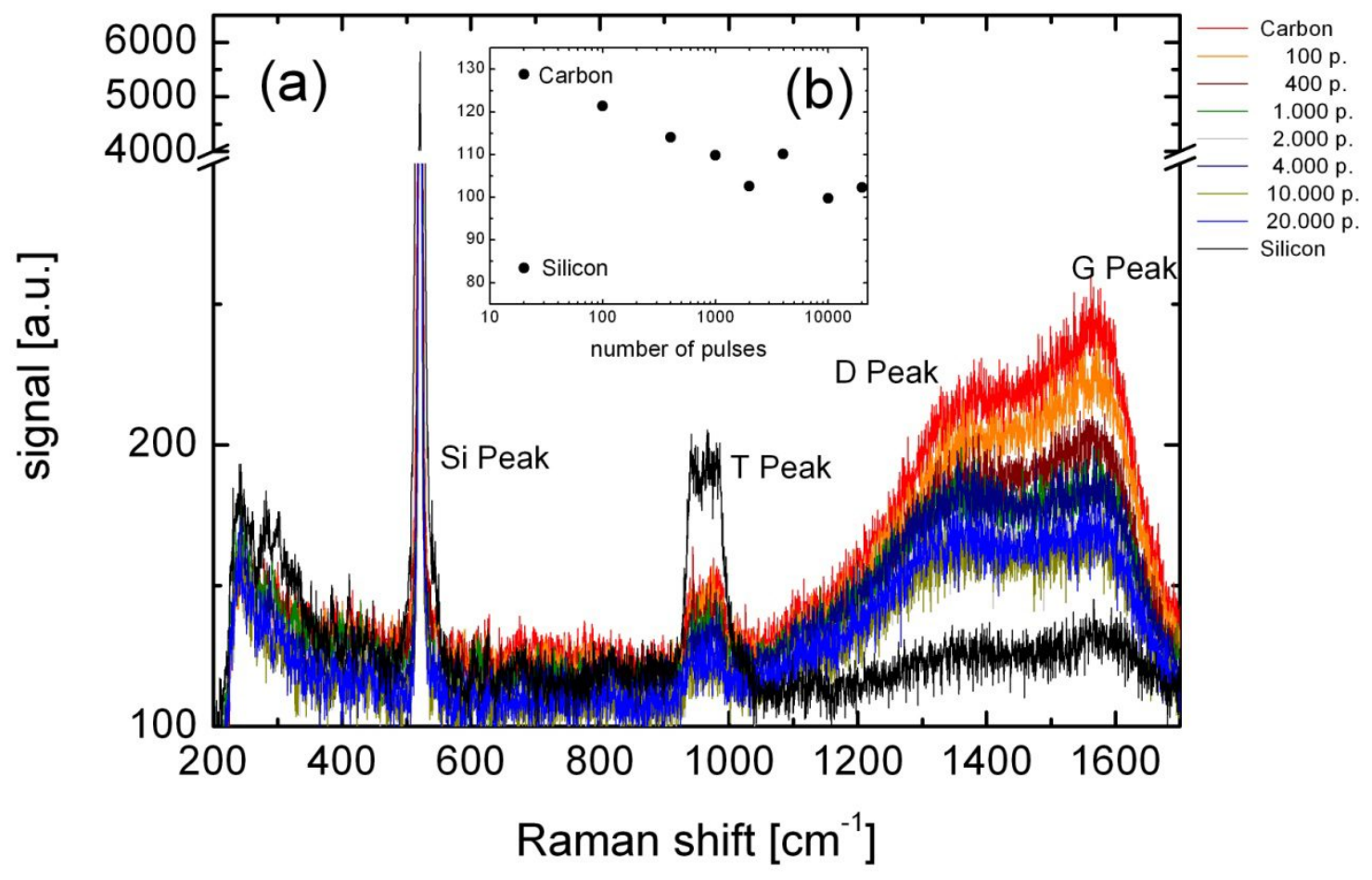

Figure 4.(a) Raman spectra for the irradiated carbon coated surface. Each spectrum indicates spot on the surface which war irradiated with a different number of Pulses except the red and black line representing unirradiated carbon and silicon spots. (b) The inlet indicates the decrease of the carbon signal (black dots) recorded with a Raman spectrometer depending on the number of pulses illuminating the investigated surface. The two data points placed at number 20 represent the Raman signal on the unirradiated carbon and the pure silicon surface, respectively 


\subsection{Discussion}

Analyzing the illuminated spots on the surface a change in the reflectivity under an optical microscope and a decrease of the carbon signal in the Raman spectra depending on the rate of illuminated pulses is observed. Surprisingly, no explicit change in the surface height is detected. A simultaneously non-thermal desorption of the carbon coating and an extrusion of the Si substrate could be supposed processes. This conclusion is reasonable due to the findings of [15] where a ablation caused by multiple laser shots below the single shot damage threshold could be observed and [13] with the findings of extrusions for a-C under illumination with fluences below $0.2 \mathrm{~J} / \mathrm{cm}^{2}$ at a wavelength of $32.5 \mathrm{~nm}$ at FLASH. Also the radiation in the present experiment with a wavelength of $21 \mathrm{~nm}$ has a transmission through the carbon coating to the silicon substrate of $50 \%$ under an angel of $\alpha=80^{\circ}$ [4]. This low intensity can also induce changes in the silicon substrate not analyzed in this investigation. Due to the flattening of the $G$ peak also a change of the order in the amorphous carbon leading to surface changes is possible.

\section{CONCLUSION}

The presented measurements indicate a change in the surface composition of carbon coated substrates under multiple pulse illumination. The reason for this finding is unclear and required more investigations. The FLASH intensities applied below the damage threshold. As a stress test for mirror coatings under multiple high energy photon pulses these findings leads to the determination that coating defects are unlikely. Anyway a critical damage of the mirrors exposed to high photon energy pulses with fluences below the damage threshold could also be expected for mirrors used under gracing incident angles. Measurements under grazing incident angles show damages in the coated surface [14, 18]. More experiments with a larger number of pulses under gracing incident angles would be favorably.

\section{ACKNOWLEDGMENT}

The authors gratefully acknowledge technical contributions by S. Eppenhoff and the mechanical workshop of the Westfälischen Wilhelms-Universität Münster. The AFM measurements were done in a fruitful teamwork with Jan-Erik Schmutz and the Profilometer was provided by NanoAnalytics. Financial support was applied by the Bundesministerium für Bildung und Forschung via Grant No. 05 KS4PMC/8.

\section{REFERENCES}

[1] Ackermann W. et al., "Operation of a free-electron laser from the extreme ultraviolet to the water window," Nat. Photonics 1, 336 (2007)

[2] Amann A. et al., "Demonstration of self-seeding in a hard-X-ray free-electron laser," Nature Photonics 6, 693 (2012)

[3] Tiedtke K. et al., "The soft x-ray free-electron laser FLASH at DESY: beamlines, diagnostics and end-stations," New J. Phys. 11, 023029 (2009)

[4] Henke B. L., Gullikson E. M., and Davis J. C. "X-ray interactions: photoabsorption, scattering, transmission, and reflection at E=50-30000 eV, Z=1-92", Atomic Data and Nuclear Data Tables Vol. 54, 181 (1993)

[5] Wöstmann M., et al,. "The XUV split-and-delay unit at beamline BL2 at FLASH," J. Phys. B 46, accepted

[6] Mitzner R. et al., "Direct autocorrelation of soft-x-ray free-electron-laser pulses by time-resolved two-photon double ionization of He," Phys. Rev. A 80, 025402 (2009)

[7] Siemer B., Hoger T., Rutkowski M., Treusch R and Zacharias H., "Desorption of ionic species from ice/graphite by femtosecond XUV laser pulses,“ J. Phys.: Condens. Matter 22, 084013 (2010)

[8] Siemer B., Olsen T., Hoger T., Rutkowski M., Thewes C., Düsterer S., Schiotz J. and Zacharias H., "Desorption of H atoms from graphite (0001) using XUV free electron laser pulses," Chem. Phys. Lett. 500, 291 (2010)

[9] Roling S. et al., "Temporal and spatial coherence properties of free-electron-laser pulses in the extreme ultraviolet regime," Phys. Rev. special topics: Accelerators and beams 14, 080701 (2011)

[10] Günther C. M. et al., "Sequential femtosecond X-ray imaging," Nat. Photonics 5, 99 (2011)

[11]Pfau B. et al., "Femtosecond pulse X-ray imaging with a large field of view," New J. Phys. 12, 095006 (2010) 
[12] Krikunova M. et al., "Ionization dynamics in expanding clusters studied by XUV pump-probe spectroscopy,” J. Phys. B: At. Mol. Opt. Phys. 45, 105101 (2012)

[13] Hau-Riege S. P. et al., "Damage threshold of inorganic solids under free-electron-laser irradiation at $32.5 \mathrm{~nm}$ wavelength," Appl. Phys. Lett. 90, 173128 (2007)

[14] Farahani Sh. D. et a., "Damage threshold of amorphous carbon mirror for 177 eV FEL radiation," Nucl. Instr. Meth. Phys. Res. Sec. A 635, S39 (2011)

[15] Juha L. et al., "Radiation damage to amorphous carbon thin films irradiated by multiple $46.9 \mathrm{~nm}$ laser shots below the single-shot damage threshold," J. Appl. Phys. 105, 093117 (2009)

[16] Richter M. et al., "Measurement of gigawatt radiation pulses from a VUV/EUV free-electron laser," Appl. Phys. Lett. 83, 2970 (2003)

[17]Ferrari A. C. and Robertson J., "Resonant Raman spectroscopy of disordered, amorphous, and diamondlike carbon," Phys. Rev. B 64, 075414 (2001)

[18] Chalupský J. et al., "Damage of amorphous carbon induced by soft x-ray femtosecond pulses above and below the critical angle," Appl. Phys. Lett. 95, 031111 (2009) 